\title{
Die Zukunft der Europäischen Union oder die Zukunft des Paradigmas der Einheit
}

\author{
Wolfgang Schmale*
}

Über die ,historische Zukunft Europas ‘ nachzudenken, stellt eine wirkliche Herausforderung dar. ${ }^{1}$ Die Formulierung ist auf anregende Weise ungewöhnlich, denn das Adjektiv , historisch" scheint eigentlich nicht zu ,Zukunft", sondern, wenn, dann zu ,Vergangenheit" zu gehören. Aber ebenso wie über die politische oder ökonomische Zukunft debattiert werden kann, ist die Frage nach der historischen Zukunft berechtigt. Im Kern geht es darum zu überlegen, ob bestimmte historische Voraussetzungen, die im Lauf der letzten Jahrzehnte oder vielleicht auch Jahrhunderte entstanden sind, auch in Zukunft Gültigkeit beanspruchen werden oder nicht. Geschichte besteht aus permanentem Wandel, mal schneller, mal langsamer, mal radikaler, mal unmerklicher, mal stehen die Kontinuitäten im Vordergrund, mal die Brüche, die ,Neuanfänge“ oder gar die ,Stunde Null', wie sie die französischen Revolutionäre 1789 gekommen sahen. Nicht nur der radikale, offensichtliche, sondern auch der weniger offensichtliche, manchmal fast unmerkliche Wandel bedarf unserer Aufmerksamkeit, wenn wir in die Zukunft blicken.

Die ,Zukunft Europas “ bedeutet faktisch die ,Zukunft der Europäischen Union“. Auch jene europäischen Länder, die derzeit nicht der Europäischen Union angehören, positionieren sich überwiegend im Verhältnis zur Union. Das gilt für eine Gruppe von Ländern, die gewissermaßen von EU-Mitgliedsländern umgeben sind oder direkt an sie angrenzen (Schweiz, Liechtenstein, Monaco, Andorra, San Marino, Norwegen, Island) und mit der Europäischen Union eng verflochten sind. Das gilt für eine zweite Gruppe von aktuellen Beitrittskandidaten (Kroatien, Türkei), mit denen bereits verhandelt wird. Das gilt für eine dritte Gruppe, für die die Frage eines künftigen Beitritts konkret diskutiert wird, auch wenn eine Vielzahl spezieller Probleme noch der Lösung harrt (Serbien, Montenegro, Albanien, Ehemalige jugoslawische Republik Mazedonien; Kosovo: bis auf Weiteres ein Sonderfall). Für die Ukraine spielt der Bezug auf die Europäische Union eine durchaus notable, wenn auch kontroversielle Rolle. Am wenigsten scheint ein Bezug zur Europäischen Union in Belarus und Moldova Aktualität zu besitzen.

Blickt man auf die Beziehungen zwischen der Europäischen Union und den Kaukasussowie zentralasiatischen Staaten, zwischen der Union und den Staaten des Nahen Ostens sowie den südmittelmeerischen Staaten (insbesondere Maghreb), dürfte es für die Länder der dritten Gruppe und die weiteren genannten auf mittlere oder längere Sicht kaum eine Alternative geben als sich der Union immer mehr anzunähern. Russland bleibt in dieser Zukunftsprojektion eine schwer zu berechnende Größe: Wird es eine attraktive Alternative zur Union für die Ukraine, Belarus, Moldova, für die Kaukasus- und zentralasiatischen Länder darstellen können und wollen? Wie wird es sich selbst im Verhältnis zur Europäischen Union sehen und definieren?

O. Univ. Prof. Dr. Wolfgang Schmale, Institut für Geschichte, Universität Wien.

1 Der Text basiert auf einem Vortrag, den der Verfasser auf der wissenschaftlichen Konferenz ,50 Jahre Römische Verträge“ im Rahmen des Panels „Die historische Zukunft Europas“ gehalten hat. Die Konferenz wurde vom Institut für Europäische Politik in Zusammenarbeit mit dem Arbeitskreis Europäische Integration und dem Centre International de Formation Européenne in Berlin durchgeführt. Angesichts der breiten, hier relevanten Literatur beschränken sich die Anmerkungen auf ein Minimum. 
Trotz dieser offenen Frage ändert sich nichts an der Feststellung, dass die ,Zukunft Europas " nichts anderes als die ,Zukunft der Europäischen Union' ist, die ,historische Zukunft" der Union wird wohl - oder übel - über die Zukunft der anderen europäischen Regionen und Staaten mitbestimmen.

\section{Das Paradigma der Einheit}

Die ,historische Zukunft` stellt ein weites Feld dar, aus dem vor allem ein Aspekt herauszugreifen ist, von dem zu erwarten ist, dass er wesentlich die Zukunft beeinflusst. Es handelt sich um das Verhältnis von ,Einheit' und ,Vielfalt', das sich in der Devise der Europäischen Union ,In Vielfalt geeint‘ niederschlägt. Nicht erst der Gipfel im Juni 2007 in Brüssel hat offenbart, wie schwierig diese Devise inzwischen in der Praxis geworden ist. Es sind immer wieder nationale Vorbehalte, die eine europäische Einigung nicht nur erschweren, sondern gelegentlich verhindern, sodass Stillstand, jedenfalls kein Fortschritt im Sinne fortschreitender Integration eintritt. Es gab in den letzten Jahren Anlässe genug - wie das „Non“ der Franzosen und das „Nee“ der Niederländer 2005 zum Verfassungsvertrag -, um Zweifel am Paradigma der europäischen Einheit aufkommen zu lassen. Öffentlich wird es kaum in Frage gestellt. Vielen Kommentatoren in den Medien war die Erleichterung anzumerken, dass auf dem Gipfel im Juni 2007 eine Lösung für das gescheiterte Verfassungsprojekt gefunden wurde, die nur im umgangssprachlichen Sinne als ,Einigung' bezeichnet werden kann - und dass eine ,Spaltung "verhindert worden sei. Angesichts der Kriegsgeschichte des europäischen 20. Jahrhunderts, des „dunklen Kontinents“, wie Mazower das Europa des 20. Jahrhunderts nannte, ${ }^{2}$ der Abermillionen Toten, der Völkermorde, des Holocaust und der riesigen materiellen Schäden, versteht es sich geradezu von selbst, dass ,Einheit" so etwas wie ein heiliges Wort darstellt. In Sachen europäischer Einheit nicht weiterzukommen, gar Rückschritte befürchten zu müssen, ruft auf dem Hintergrund des ,dunklen 20. Jahrhunderts', wie Mazowers Titel abgeändert werden könnte, negative Emotionen hervor. ,Europäische Einheit' steht für ein gelungenes Friedensprojekt, für Wohlstand, für eine Vielzahl von Freiheiten, für Sicherheit, die aus der Allgemeingültigkeit von Demokratie, Rechtsstaatlichkeit und Menschenrechten erwächst. Dies erklärt mehr als hinreichend die emotionale Strahlkraft von ,Einheit".

Und ist es nicht so, dass der Ruf nach europäischer Einheit uns seit rund 700 Jahren begleitet? Ist er nicht der am meisten vertraute Ruf seit den politischen Europaplänen eines Pierre Dubois um 1306, eines Georg von Podiebrad (1464), eines Sully (1638), eines Abbé de Saint Pierre (1713), eines Kant (1795), eines Victor Hugo (1849), eines Aristide Briand (1930), eines Altiero Spinelli im Gefängnis von Ventotene (1941), der Geschwister Scholl (1942)?

Das Paradigma der Einheit zählt zum Inventar zentraler politischer Paradigmen in Europa. Ob nun - historisch - die Einheit der Kirche oder die europäische Universalmonarchie des Kaisers (des Heiligen Römischen Reiches deutscher Nation), die Einheit der National-Monarchien wie Portugal (seit der ersten Hälfte des 15. Jahrhunderts), Spanien (seit 1492), Frankreich und England (seit dem Mittelalter), die Einheit der (Stadt-)Republiken wie Venedig beziehungsweise die Vereinigten Niederlande oder die Einheit des Nationalstaats (besonders seit dem 19. Jahrhundert) gemeint ist, die jeweiligen politischen Gebilde werden grundsätzlich mittels eines Bildes von Einheit erfasst. Im Spätmittelalter und der Frühen Neuzeit wurden als Bilder der Einheit allegorische Figuren eingesetzt. Die Füh-

2 Mark Mazower: Dark Continent: Europe's Twentieth Century, London 1998. 
rungsfiguren wie Papst, Kaiser, Könige oder Königinnen stellten gleichfalls Personifizierungen von Einheit dar. Auch Herrschaftsinsignien oder andere, weitere Bilder wie der Quaternionenadler des Heiligen Römischen Reiches ${ }^{3}$ sprachen die Bild-Sprache der Einheit. Die Nationen und Nationalstaaten des ausgehenden 18. bis 20. Jahrhunderts wurden - ganz in der Tradition der Frühen Neuzeit - durch Personifizierungen wie die Germania, Francia (oder Marianne), Hispania oder durch mythische Heldenfiguren, zumeist vermeintliche oder auch ,echte ' Widerständler/innen gegen die römische Herrschaft (Hermann in ,Deutschland', Vercingetorix in Frankreich, Viriathus in Spanien, Civilis in den Niederlanden, Boadicea in England, etc.) repräsentiert. ${ }^{4}$ Die Beliebtheit der Personifizierungen als Bilder der Einheit ist eng verbunden mit der fundamentalen Vorstellung von ,Körper', der ,Wirklichkeit' und Metapher von Einheit schlechthin im Mittelalter und in der Neuzeit (bis weit in das 20. Jahrhundert hinein).

Dasselbe Paradigma und Bild der Einheit wurde auch auf Europa angewandt. Das Bild vom ,Haus Europa' stammt aus dem 15. Jahrhundert, der Humanist (und spätere Papst) Enea Silvio Piccolomini benutzte es (1454), um die Einheit der europäischen Monarchien angesichts der Türkengefahr zu beschwören. Seit dem 16. Jahrhundert waren verschiedene und zahlreiche Varianten der Erdteilallegorie Europas im Umlauf, die Europa sehr eingängig als Christliche Republik repräsentierten. Im späten 17. und vor allem seit dem 18. Jahrhundert stellte die Erdteilallegorie Europa als Kultur (im Singular!) dar, im Grunde ein Synonym für Einheit. Die Kulturgeschichtsschreibung des 18. Jahrhunderts stellte alle Parameter bereit, derer es bedurfte, um Europa als einheitliche Kultur zu begreifen. ,Europäische Kultur' im Singular wurde mit der Vorstellung vom ,politischen System`Europas korreliert. Das gemeinsame war der Systembegriff: Kultur als System, die Beziehungen der europäischen Staaten zueinander als System. Das politische System wurde zumeist als Balance of Power bezeichnet, aber es hielt sich die Rede von der Christlichen Republik noch bis weit in das 18 . Jahrhundert. Die Idee einer Heiligen Allianz, die der russische Zar 1815 aus Anlass des Wiener Kongresses lancierte, und die zunächst zwischen Russland, Österreich und Preußen geschlossen, später erweitert wurde, setzte die Idee der Christlichen Republik fort. Um die Einheit Europas auszudrücken - als politische Zielvorstellung oder als Tatsachenfeststellung, die sich dann zumeist auf Europa als Kultur bezog - blieben im 19. Jahrhundert die Erdteilallegorien im Gebrauch, aber es wurden auch neue Zielbegriffe geschaffen wie etwa - in Anlehnung an die USA, dem großen Vorbild für die Möglichkeit, großer' Demokratien ,Vereinigte Staaten von Europa' 5

Im 20. Jahrhundert wurde unter dem Eindruck zunächst des Ersten Weltkrieges Europäische Einheit genauer und praktischer gedacht. Die Vorstellung von einem föderal verfassten europäischen Staat, die in der Tradition des Begriffs ,Vereinigte Staaten von Europa'steht, übersetzt das Paradigma der Einheit am konkretesten. Die Idee hatte auch unter vielen Widerstandsgruppen im Zweiten Weltkrieg Anhänger. Die Bedeutung der Föderalisten nach 1945 ist hoch anzusetzen, selbst wenn keines der realisierten Projekte wie die Europäische Gemeinschaft für Kohle und Stahl (EGKS) die föderalistische Konzeption Europas umsetzte. Europäische Föderalisten finden sich auch heute im Europäischen Parlament, sie tra-

Rainer A. Müller (Hrsg.): Bilder des Reiches, Sigmaringen 1994.

4 Details bei Hagen Schulze: Staat und Nation in der europäischen Geschichte, 2. Aufl., München 1995, S. 109.

5 Die Bildsprache der europäischen Einheit ist genauer untersucht bei: Wolfgang Schmale: Europapropaganda, in: Rainer Gries/Wolfgang Schmale (Hrsg.): Kultur der Propaganda, Bochum 2005, S. 285-304. Die Idee europäischer Einheit ist am besten dargestellt bei: Derek Heater: Europäische Einheit - Biographie einer Idee. Übersetzt und annotiert von Wolfgang Schmale und Brigitte Leucht, Bochum 2005 (Englisch: The Idea of European Unity, London 1992). 
ten im Konvent, der den Entwurf für einen Verfassungsvertrag ausarbeitete, auf, es gibt föderalistische Vereinigungen. ${ }^{6}$

Der nach 1945 konkret beschrittene Weg wurde mit anderen, aber nicht weniger auf Einheit zielenden, Leitbegriffen beschritten: Der wichtigste wurde ,Integration ' ${ }^{7}$ Es verdient festgehalten zu werden, dass dieser Begriff ähnlich wie das Paradigma ,Vereinigte Staaten ‘ aus den USA nach Europa kam. Paul Hoffman, Administrator der amerikanischen Marshallplan-Verwaltung, führte ihn in einer Rede am 31. Oktober 1949 vor der, Organization of European Economic Cooperation' (OEEC, Vorgängerin der OECD) ein. ,Integration “ bildete fortan ein semantisches Feld mit Gemeinschaft beziehungsweise Union. Integration gehört zur Kategorie der Prozess-Begriffe, Integration ist ein Ziel, das am Ende eines mehr oder weniger langen Entwicklungsprozesses steht und zu dessen Erreichen bestimmte Maßnahmen gemeinsam getroffen und umgesetzt werden. Die Eckpunkte des im Allgemeinen Integration genannten Prozesses wurden im EWG-Vertrag (25. März 1957) in der Präambel formuliert. Einige dieser Eckpunkte, die zugleich ein semantisches Feld ergeben, seien in aller Kürze in Erinnerung gerufen (Begriffe der Einheit sind kursiv gesetzt, Begriffe der Integration sind unterstrichen): ,die Grundlagen für einen immer engeren Zusammenschlu $\beta$ der eu-

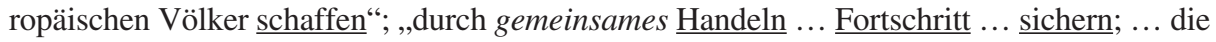
Europa trennenden Schranken beseitigen“; ,zur Beseitigung der bestehenden Hindernisse (ist) ein einverständliches Vorgehen erforderlich“; ,ihre Volkswirtschaft ... einigen und deren harmonische Entwicklung ... fördern“; ,gemeinsame Handelspolitik zur fortschreitenden Beseitigung der Beschränkungen“; „,durch diesen Zusammenschlu $\beta$ ihrer Wirtschaftskräfte Frieden und Freiheit zu wahren und zu festigen, und mit der Aufforderung an die anderen Völker Europas, die sich zu dem gleichen hohen Ziel bekennen, sich diesen Bestrebungen anzuschließen“; etc. Der heute im Vordergrund stehende Unionsbegriff wurde 1972 in Paris (Europäischer Gipfel) aufgewertet, als die „Umwandlung“ der „Gesamtheit der Beziehungen der Mitgliedstaaten in eine Europäische Union“ zum wichtigsten Ziel erklärt wurde. Im sogenannten Spinelli-Bericht des Europäischen Parlaments von 1984 wurde versucht, den Unionsbegriff in der Tradition der europäischen Föderalisten verfassungsrechtlich zu konkretisieren. Durchgesetzt hat sich die Formulierung weiterer gemeinsamer Ziele, die sich vorrangig auf Demokratie und Menschenrechte beziehen, sowie der Zusammenbau von bisheriger Integration in Gestalt supranationaler Institutionen mit Elementen des Intergouvernementalismus. ${ }^{8}$ Nach Außen wird die Einheit durch im Grunde typisch nationalstaatliche Symbole vermittelt: Flagge, Hymne, Europatag (analog zu den Nationalfeiertagen). Hinzukommt eine (schon zitierte) Devise - wohingegen heute keineswegs alle Nationalstaaten über eine Devise verfügen (Frankreich allerdings besitzt gleich zwei Leitsprüche aus der jakobinischen Phase der Französischen Revolution: La République une et indivisible; Liberté, Egalité, Fraternité).

Diese heuristisch zusammengestellten Fakten belegen die Verbindlichkeit des Paradigmas der Einheit in Bezug auf die Europäische Union und ihre Vorgängerinnen. Ergänzen lässt sich noch die Bedeutung des Paradigmas der ,Mitte', denn jede Einheit hat in der euro-

6 Die umfassendste Dokumentation ist zu finden in: Walter Lipgens (Hrsg.) bzw. Walter Lipgens/Wilfried Loth (Hrsg.): Documents on the History of European Integration: 4 Bände, Berlin/New York 1985-1991. Zum Europäischen Verfassungskonvent: Peter Becker/Olaf Leiße: Die Zukunft Europas. Der Konvent zur Zukunft der Europäischen Union, Wiesbaden 2005; Daniel Göler: Deliberation - Ein Zukunftsmodell europäischer Entscheidungsfindung? Analyse der Beratungen des Verfassungskonvents 2002-2003, Baden-Baden 2006.

7 Zur Begriffsgeschichte siehe Ludolf Herbst: Die zeitgenössische Integrationstheorie und die Anfänge der europäischen Einigung 1947-1950, in: Vierteljahreshefte für Zeitgeschichte 34/1986, S. 161-205.

8 Werner Weidenfeld/Wolfgang Wessels (Hrsg.): Europa von A bis Z, 10. Aufl., Baden-Baden 2007, S. 185. 
päischen Vorstellungswelt auch eine Mitte oder ein Herz. In der Frühen Neuzeit, ja bis ins 20. Jahrhundert in bestimmten Fällen, wurde besonders Böhmen als der „Bauchnabel Europas" bezeichnet, mithin als Europas Mitte. Seit 1989 kennen wir einen angeregten Wettbewerb um die Position, Europas Mitte zu sein. Etwas pseudowissenschaftlich wird die Mitte geographisch begründet; je nachdem, von wo aus gemessen wird - dem Graben zwischen der amerikanischen und der eurasischen Kontinentalplatte, der (unter anderem) unter Island verläuft, oder von der französischen Atlantikküste und so weiter - befindet sich die vermeintliche geographische Mitte Europas in Frankreich, in Deutschland, in der Ukraine in der Nähe von Lemberg oder in Litauen (etc.). Andere reklamieren für sich, im „Herzen Europas“ zu liegen. ${ }^{9}$ Die Rede von einer „Seele Europas“ stellt im Grunde nichts anderes als eine weitere Variante des Paradigmas der Mitte dar. Nicht zufällig rankt sich um den von Metternich geprägten Begriff ,Mitteleuropa“ (heute im Englischen ,Central Europe', im Französischen ,Europe centrale', und als Entlehnung hieraus neuerdings im Deutschen auch ,Zentraleuropa') eine reiche Literatur. ${ }^{10}$

Beim Versuch, das komplexe Gebilde namens Europäische Union, wie es der EU-Vertrag (vorerst in der Fassung von Nizza, demnächst vielleicht in der Fassung von Lissabon) façonniert, darzustellen, wird gern auf das Wort und das Bild der, Tempelkonstruktion` zurückgegriffen. ${ }^{11}$ Das wiederum steht in der Tradition der Metapher vom ,europäischen Haus', die im 15. Jahrhundert benutzt wurde, die in bebilderten Broschüren zur Popularisierung des Marshallplanes vorkam und die vom letzten Präsidenten der Sowjetunion Michail Gorbatschow eingesetzt wurde. Haus, Gebäude, Tempel, auch sie sind Metaphern der Einheit.

\section{Vielfalt}

Wo liegt das Problem, wenn das alles so schön passt? Es liegt in der Praxis, in dem Widerspruch zwischen Einheitsideal und Tatsächlichkeit der europäischen Vielfalt, die oft schlicht eine Vielheit darstellt, die nicht unter, ein Dach`zu bringen ist. Außerdem scheint der Wunsch nach mehr Einheit zunehmend weniger einheitsfähig zu sein. Schon lange gibt es für einzelne Mitgliedsländer Sonderregelungen oder das ,Opting-out' bei einzelnen Regelungen, wie jüngst im Juni auf dem Brüsseler Gipfel. Es bestehen durchaus grundsätzliche Unterschiede zwischen den Idealen des Vereinigten Königreichs, die nicht wirklich über die engeren Zielsetzungen des seinerzeitigen EWG-Vertrages hinausgehen, und jenen anderer Länder, die den Weg zu einer ,immer engeren Union“ weiter beschreiten wollen. Ohne genauer hinzuschauen, können hierzu die 18 Länder gezählt werden, die den Verfassungsvertrag bereits per Parlamentsbeschluss oder per Volksbefragung ratifiziert hatten.

Im Diskurs der Europäischen Union wird das Verhältnis von Einheit und Vielfalt durch zwei Grunddenkmuster ausgedrückt, die die Soziologin Gudrun Quenzel am Beispiel der (EU-)europäischen Identitätskonstruktion herausgearbeitet hat. Es handelt sich einerseits um die „Baumstruktur“, andererseits um das „Netzwerk“.12 Das Denkmuster „Baumstruktur“ setzt eine ,starke vorgängige Einheit voraus“. ${ }^{13}$ Hier lässt sich das oben skizzierte Paradigma der Einheit einordnen. Politische Gebilde werden in Europa als Einheit gedacht. Das

9 Georg Kreis: Europa und seine Grenzen, Bern 2004, S. 42-46.

10 Vgl. statt vieler Barbara Breysach (Hrsg.): Europas Mitte - Mitteleuropa - europäische Identität?, Berlin 2003.

11 Siehe statt vieler zur Illustration die entsprechende Zeichnung in Weidenfeld/Wessels (Hrsg.): Europa von A bis Z, 2007, S. 188.

12 Gudrun Quenzel: Konstruktionen von Europa. Die europäische Identität und die Kulturpolitik der Europäischen Union, Bielefeld 2005, hier besonders Kapitel 4.6 (Strategien europäischer Identitätskonstruktionen).

13 Quenzel: Konstruktionen, 2005, S. 201. 
erscheint wie eine Selbstverständlichkeit, ohne es tatsächlich zu sein. Die wenigsten politischen Gebilde in der europäischen Geschichte stellten in der Realität eine Einheit dar. Aber auch die Vorstellung von europäischer Kultur (im Singular) findet in diesem Denkmuster Platz: Die erwähnte Kulturgeschichtsschreibung der Aufklärung schuf diese Vorstellung einer einheitlichen europäischen Kultur, allen voran Voltaire mit seinem umfassenden ,Essai sur les mœurs et l'esprit des nations (...) ${ }^{\text {(1756) }}{ }^{14}$ die allerdings im 19. und 20. Jahrhundert in zweierlei Hinsicht aufgebrochen wurde. Zum einen wurde der Begriff der europäischen Kultur auf Westeuropa reduziert, zum anderen wurde er im Rahmen rassenkultureller Lehren pervertiert und zerstört. Sofort nach 1945 wurde hingegen ,kulturelles Erbe' (cultural heritage) zu einem Schlüsselwort, das über den Brüsseler Pakt von 1948 Eingang in viele spätere auch EG- und EU-Dokumente fand. Das ,kulturelle Erbe' ist ein positiv konnotierter Begriff, der sich ursprünglich besonders auf Demokratie und Rechtsstaatlichkeit als das eigentlich Europäische - im Gegensatz zur Nazi-Barbarei - bezog. Insbesondere in der (EU-) europäischen Identitätskonstruktion wurde an dieser Denkfigur zentral festgehalten, sodass sie zum Denkmuster wurde, ausgedrückt auch konkret im Bild des Baumes. ${ }^{15}$

Zunehmend behauptet sich ein zweites Denkmuster, nämlich ,Netzwerk', im EU-Diskurs. Es bezieht sich einerseits auf die Vernetzung von Individuen im europäischen Raum zu einer Kommunikationsgemeinschaft wie auch, andererseits, auf die Vernetzung von Institutionen, zum Beispiel in den Bereichen von Kunst und Kultur (Museen und so weiter), von Bildung (Schulen) oder Wissenschaft (Universitäten). Die Ermöglichung von Netzwerken im Sinne der Schaffung von Kommunikationsgemeinschaften geht teilweise über Europa hinaus (vergleiche Erasmus Mundus-Programme, etc.). Nach Quenzel besteht der Hauptzweck der Vernetzung zu Kommunikationsgemeinschaften in der „Europäisierung von nationalen Kulturgütern“. 16

Wie ,Integration“ gehört ,Europäisierung' zu jenen Prozessbegriffen, die zielgerichtete Entwicklungen hin zu Einheit zusammenfassen. Es liegt in der Konsequenz des Einheitsparadigmas, dass die durch die vielen Nationen und national geprägten Kulturen repräsentierte Vielfalt und nicht selten konfliktträchtige Vielheit durch Europäisierung ihres Konfliktpotenzials enthoben werden soll. Quenzel schlussfolgert: „Die Europäische Union versucht damit über die Erzeugung eines gemeinsamen (sic!) Konsenses zu integrieren und nicht über die Etablierung einer produktiven Konfliktkultur, in der gerade über Auseinandersetzung eine erhöhte Partizipationsbereitschaft sowie ein Gemeinschaftsgefühl erzeugt werden." 17

So mag die Union ,Union“ heißen, doch dieser Einheit suggerierende Name geht beinahe verloren, wenn die juristischen, politikwissenschaftlichen oder auch historischen, Übersetzungen' von Union in andere deskriptive Begriffe wie ,Mehrebenensystem` oder, institutionalisiertes Verhandlungssystem‘ unter die Lupe genommen werden.

14 François-Marie Arouet, gen. Voltaire: Essai sur les mœurs et l'esprit des nations et sur les principaux faits de l'histoire depuis Charlemagne jusqu'à Louis XIII. Introduction, bibliographie, relevé de variantes, notes et index par René Pomeau, 2 Bände, Paris 1963. Zur Europa-Historiographie vgl. nunmehr: Heinz Duchhardt/ Małgorzata Morawiec/Wolfgang Schmale/Winfried Schulze (Hrsg.): Europa-Historiker. Ein biographisches Handbuch, 3 Bände, Göttingen 2006-2007. Als gerafften Überblick über die Europa-Historiographie der Neuzeit siehe Wolfgang Schmale: Europa als Topos in der Geschichtsschreibung, in: Georg Michels (Hrsg.): Auf der Suche nach einem Phantom? Widerspiegelungen Europas in der Geschichtswissenschaft, Baden-Baden 2003, S. 45-67.

15 Vgl. das Plakat des europäischen Geschichtsbaumes: Britta Orgovanyi-Hanstein: Geschichtsbaum Europa (Plakat), 4. Aufl., Frankfurt 2003.

16 Quenzel: Konstruktionen, 2005, S. 204.

17 Quenzel: Konstruktionen, 2005, S. 201. 
Verfassungsgeschichtlich stellt ,Union“ eine häufig gewählte Bezeichnung dar, die aber verfassungsrechtlich nicht exakt oder einheitlich definiert ist - wenn überhaupt. In der Geschichte gab es mancherlei Unionen wie die polnisch-litauische (1569 besiegelt), die Protestantische Union (1608), die Sächsisch-Polnische Union (1697), die Schwedisch-Finnische und die Dänisch-Norwegische Union beziehungsweise Schwedisch-Norwegische Union (1814-1905), oder, um ein jüngeres Beispiel zu nennen, die ,Union française ' von 1946, alles Zusammenschlüsse, die keinem bestimmten Rechts- beziehungsweise Verfassungsmodell folgten. Zweifellos steckt der Begriff ,Union“ auch im , united ' der ,United States of America' oder in ,United Kingdom', aber beides sind föderale Nationalstaaten, selbst wenn hier in Bezug auf das United Kingdom Einschränkungen (Schottland, Nordirland, Wales) angebracht sind. ,Union" versteckt sich in ,United Nations', in gewisser Hinsicht auch in der Vorgängerorganisation, dem Völkerbund (League of Nations). In beiden Fällen handelt es sich aber nicht um politische Gebilde im Sinn von Staat. Im Grunde ist daher ,Union “ ein definitorisch kaum besetzter Begriff. Der nunmehr gewesene EU-Verfassungsvertragsentwurf lieferte - entgegen der Überschrift von Titel 1 („Definition und Ziele der Union“) - im Übrigen keine Definition von ,Union“.

Das Paradigma der Einheit läuft in letzter Konsequenz auf einen europäischen Staat hinaus, der aber nicht einmal von den Befürwortern der ,immer engeren Union“ als Ziel konkret formuliert wird. Das wäre zu konfliktträchtig, aber auch so denkt kein Mitgliedsland ernsthaft an diese Möglichkeit. Der Kern des Problems liegt in der Vitalität des politischen Modells des Nationalstaats. Dies programmiert die Konflikte mit der Ausrichtung der Europäischen Union am Paradigma der Einheit vor. Lässt man nur die europäischen Gipfel seit Nizza im Dezember 2000 bis Brüssel im Juni 2007 Revue passieren, so wird deutlich, dass auf gewisse Weise die Stunde der Wahrheit gekommen ist, wobei ,Stunde ' durchaus als Metapher für ein oder auch mehrere Jahre steht. Darauf kommt es nicht an - früher oder später sind weitreichende Entscheidungen zu treffen.

Die Vitalität des Nationalstaats (und der nationalen Kulturen) verdankt sich paradoxerweise dem europäischen Integrationsprozess selbst, wie es Alan S. Milward in , The Rescue of the Nation State' nachgewiesen hat. ${ }^{18}$ Der moderne, zunächst west-europäische Nationalstaat hat im Zuge dieser neuen Chance nach 1945 viele seiner unangenehmen Charakterzüge verloren. Im Großen und Ganzen kann man dazu (zu den verlorenen Charakterzügen) den Nationalismus rechnen. Das gilt für die einzelnen Staaten unterschiedlich, es gab Beispiele eines kriegerischen Nationalismus im ehemaligen Jugoslawien. Im Allgemeinen dreht es sich um Patriotismus und Nationsverbundenheit, nicht um Nationalismus. Das bedeutet nicht, die rechts-radikalnationalen Szenen, die es in allen europäischen Ländern gibt, zu übersehen. Und es bedeutet nicht, die Augen vor der Instrumentalisierung nationaler Gefühle in Wahlkampfzeiten zu verschließen, die je nach Partei und Spitzenkandidat(in) in Nationalismus abgleitet. Endgültig gebannt ist Nationalismus nicht.

Der Nationalstaat hat viele der historischen Grenzziehungen, für die er stand, aufgegeben: Er schützt heute - nach Innen - rechtlich ethnische, sexuelle, kulturelle, religiöse und andere Formen von Diversität. Nach Außen - im Rahmen des Schengen-Abkommens, dem noch nicht alle EU-Staaten beigetreten sind - sind die Grenzen weitgehend offen. Die Metaphern von Baum und Netz passen auf ihn ebenso wie auf die Europäische Union. Der europäische Nationalstaat heute ist ein Rechtsstaat, er hat eine demokratische Verfassung, er wahrt und garantiert seinen Bürgerinnen und Bürgern Grund- und Menschenrechte sowie soziale und Bildungsrechte. Man muss es sich immer wieder vor Augen halten, dass diese

18 Alan S. Milward: The European Rescue of the Nation-State, London 1994. 
Feststellung in ihrer allgemeinen Form für Westeuropa erst seit 1945/1948, für Ostmitteleuropa erst in Folge der Wende von 1989/90, für manche Staaten erst seit ganz wenigen Jahren - und für manche immer noch nicht - gilt!

Seine Vitalität rührt zum Teil daher, dass die Europäische Union und ihre Vorgängerinnen einen Rahmen zur Verfügung stell(t)en, in dem es einerseits erforderlich, andererseits eben möglich war, als national definierte Interessen in der jeweiligen Mitgliedergemeinschaft zu artikulieren und von den anderen ganz oder auch modifiziert anerkennen zu lassen. ${ }^{19}$ Vergleicht man diese Methode mit der Art und Weise, wie sich die Nationalstaaten, grob gesagt, zwischen 1848 und 1945 gegenübertraten, so ist die fundamentale Veränderung offensichtlich. Verschiedene Prinzipien, die in der Europäischen Union Gültigkeit haben, wie das Subsidiaritätsprinzip, stützen bewusst die Rolle der Nationalstaaten. Es herrscht Konsens, dass Vieles besser, vor Ort', sprich im einzelnen Staat, auf regionaler oder auch auf kommunaler Ebene, statt einheitlich auf europäischer Ebene zu regeln ist.

Die Anerkennung supranationaler Institutionen bedeutete im Ergebnis nicht den Anfang der Abschaffung des Nationalstaats, sondern ist als Teil seiner Modernisierung und phänotypischen Transformation zu werten.

Die Vordenker eines einheitlichen Nachkriegseuropas in den Widerstandsgruppen des Zweiten Weltkriegs und viele Föderalisten bedauerten zutiefst, dass die Nachkriegsordnung erneut auf der Basis von Nationalstaaten geschaffen wurde. Aber wäre 1945 die Schaffung eines europäischen, föderalen Staates wirklich machbar gewesen? Daran sind gründliche Zweifel erlaubt. So oder so: Der europäische Nationalstaat ist ein Faktum, ein veränderliches zwar, aber ein Faktum, und was unter europäischer ,Einheit' verstanden werden kann, findet seine Grenze in diesem Faktum. Das hat die Europäische Union in ein gewisses Dilemma gebracht, weil im Nationalstaat das Paradigma der Einheit gilt - so wie es auch für die europäische Einheit idealistisch gefordert wurde und wird. Es handelt sich gewiss nicht um eine Situation des Entweder-Oder, aber es geht um den künftigen Modus vivendi.

\section{Das Paradigma des Netzwerks}

Zum Paradigma der Einheit eine Alternative zu finden, die die wesentlichen Ziele der Europäischen Union, wie sie sich im Lauf von 50 Jahren entwickelt haben, garantiert, ist gewiss keine Kleinigkeit, aber lohnt die Mühe. Es wird sich dabei um eine tiefergehende und längere Debatte handeln, die im Ergebnis neue pragmatische Weichenstellungen ermöglichen wird.

Die Reform des Europäischen Vertrages, der dann vielleicht als Vertrag von Lissabon in die Geschichte eingehen wird, steht noch ganz in der Logik des Einheitsparadigmas, während der (gescheiterte) Verfassungsvertrag nicht nur in der Logik dieses Paradigmas gestanden hatte, sondern es für einige Mitglieder der Europäischen Union mit diesem Paradigma offenkundig zu weit getrieben hatte. Die voraussichtliche Reform wird mehr Einheit erbringen, wobei hier nur auf das Prinzipielle zu sehen ist, während Details und allfällige Sonderregelungen für einzelne Länder außer Acht bleiben können, da sie das Prinzipielle nicht aushebeln: Rechtsverbindliche Geltung der EU-Grundrechtecharta, mehr Einheit nach Außen durch die Einführung eines EU-Außenministers (Hoher Vertreter der Europäischen Union für Außen- und Sicherheitspolitik) und einer nur noch alle zweieinhalb Jahre wechselnden EU-Ratspräsidentschaft, Wahl des Kommissionspräsidenten durch das Europäische Parlament, mehr Kompetenzen für das Parlament, noch mehr Mehrheitsentscheide als bisher.

19 Vgl. dazu jetzt die gegenwartsbezogene Studie: Stefanie Bailer: Nationale Interessen in der Europäischen Union. Macht und Verhandlungserfolg im Ministerrat, Frankfurt 2006. 
Nehmen wir dies als Tatsachenfeststellung und bewerten es ganz nüchtern im Licht der europäischen Integrationsgeschichte. Das an europäischer Einheit Erreichte (hier immer einschließlich der $2007 \mathrm{zu}$ beschließenden Vertragsreform gedacht) ist wahrhaftig nicht wenig. Das meiste von dem, was an gemeinsamen europäischen Institutionen unter verschiedenen Namen - und natürlich bei anderen historischen Kontexten - in den Europaplänen seit mehreren Jahrhunderten in Vorschlag gebracht worden ist, wurde realisiert. Dies geschah nicht unbedingt in der Absicht oder im Bewusstsein, diese politischen Pläne nun endlich zu erfüllen, selbst wenn nicht vergessen werden sollte, dass die Politiker, die die europäische Einigung nach dem Zweiten Weltkrieg auf den Weg brachten, doch über soviel historische Bildung verfügten, dass sie die Vorschläge eines Sully (oder Heinrichs IV., dem Sully selber seinen Plan in den Mund geschoben hatte), eines William Penn, eines John Bellers, eines Kant, eines Saint-Simon, ganz zu schweigen von den Europäisten der ersten Hälfte des 20. Jahrhunderts, kannten..$^{20}$

Angesichts dieser gewiss historischen Leistung darf gefragt werden, ob mehr europäische Einheit strukturell möglich ist. Die Antwort lautet, dass realistischer Weise entscheidend mehr nicht erwartet werden kann, weil dieses ,Mehr' dann tatsächlich die historische Überwindung des Nationalstaats in Europa und die Errichtung eines unitarischen europäischen Staats bedeuten würde. Das kann derzeit aber aufgrund der oben kurz inhaltlich ausgeführten Modernität, Vitalität und Funktionalität des Nationalstaats nicht ohne Risiko für das Erreichte auf die Tagesordnung gesetzt werden. Ob dies in einigen Jahrzehnten möglich sein wird, ist schwer vorherzusagen.

Wenn derzeit im Großen und Ganzen das an europäischer Einheit erreicht ist, was historisch erreicht werden konnte - der Beitritt einiger weiterer Voll-Mitglieder ist hierin bereits , eingerechnet “ - heißt das dennoch nicht, dass nunmehr Stagnation eintreten wird. Seit Längerem existieren Vorschläge, die implizit von diesem hier deutlich formulierten Befund ausgehen: Es wurde Vertiefung der Integration statt weiterer Beitritte gefordert. Es ist die Rede von einem Europa der zwei Geschwindigkeiten; ins Auge gefasst wird die bewusste Fortsetzung einer bereits geübten Methode, nämlich dass die Mitglieder, die in einem bestimmten Feld mehr Einigung wollen als andere, dieses Mehr umsetzen. Dies wurde bezüglich des kommenden Reformvertrages ausdrücklich bekräftigt (mögliche engere Zusammenarbeit nur eines Teils der Mitglieder bei Justiz, Polizei und in der Sozialpolitik). Zur Währungsunion und zum Schengen-Abkommen würden weitere Bereiche kommen, was in der Konsequenz auch zu mehr als ,zwei Geschwindigkeiten“ führen könnte. Statt Vollmitgliedschaft werden alternative Modelle wie ,privilegierte Partnerschaft' (ein sehr unglücklich gewählter Begriff) stark gemacht; es existiert das Instrument der strategischen Partnerschaft (aktuell: EU-Brasilien).

Im Innern der Union sind unterschiedliche politische Kommunikationsgemeinschaften auszumachen: bilaterale (zum Beispiel die regelmäßigen deutsch-französischen Regierungskonsultationen), trilaterale (das ,Weimarer Dreieck' aus Frankreich, Deutschland und Polen) oder die regelmäßigen Treffen der Präsidenten Österreichs und anderer ostmitteleuropäischer Länder, die ehemals zur Habsburgermonarchie gehört hatten. Der Europäische Wirtschaftsraum (EWR) konstituiert eine Kommunikationsgemeinschaft, die über die Europäische Union hinausreicht. Es gäbe zahlreiche weitere Beispiele. Bei der Vorbereitung von Entscheidungen in der Union spielen komplexe Kommunikationsflechtwerke eine Rolle, in die zusätzlich zu den verfassungsmäßig involvierten europäischen Institutionen Lobbyverei-

20 Heater: Europäische Einheit, 2005, geht in den jeweiligen Kapiteln auf das Nachleben der besprochenen Pläne ein. 
nigungen, Regionen, Bundesländer, Städte, NGOs, Parteien, Parlamente, Gewerkschaften, Wirtschaftsverbände und so weiter eingebunden sein können. Allein schon das institutionelle System konnte bereits 1994 von Heinrich Schneider als ,hybrid“ bezeichnet werden. ${ }^{21}$

Was wie ein unkoordiniertes Sammelsurium politischer Praktiken daher kommt, lässt sich durchaus systematisch denken. Heinrich Schneider schlug den Begriff der „Ordnungsverfassung“ im Kontext einer „Föderalen Union neuen Typs“ vor. ${ }^{22}$ Wolfgang Wessels sprach als mögliche künftige Verfassungsform der Europäischen Union von „fusioniertem Föderalstaat“", wo „,staatliche Akteure mehrerer Ebenen gemeinsam und in variierenden Graden von Intensität an der Vorbereitung, Herstellung, Durchführung und Kontrolle allgemein verbindlicher Entscheidungen zum Einsatz legislativer und budgetärer Handlungs- und Steuerungsinstrumente beteiligt [sind] und dabei spezifische Verfahren gemeinsamer Problemverarbeitung (entwickeln).“23

In jüngeren Arbeiten wird in der Folge ähnlicher Zustandsbeschreibungen der Begriff der Kohärenz, um deren Herstellung es geht, in den Vordergrund gerückt. ${ }^{24}$ Die verschiedenen politikwissenschaftlichen und verfassungsrechtlichen Zustandsbeschreibungen der praktischen und faktischen Verfassung der Europäischen Union, die hier mitnichten ausführlich referiert werden können, sind in der Soziologie und in der Geschichtswissenschaft als Beschreibungen bestimmter historischer und gegenwärtiger Phänomene bekannt, die mit dem Begriff des ,Netzwerks ' belegt werden, da es schon längst nicht mehr nur um staatliche Akteure geht. ${ }^{25}$ Diese Feststellung gilt ebenso für den Nationalstaat. Bezeichnungen wie ,partizipative Demokratie' oder gar ,postparlamentarische Demokratie' beziehen sich auf den Umstand, dass politische Akteure außerhalb der klassischen drei Gewalten immer mehr Gewicht erhalten. Zivilgesellschaftliche Akteure sind hierbei besonders hervorzuheben. Als symptomatisches ,nationales ' Beispiel postparlamentarischer Tendenzen kann die öffentliche Kritik dreier türkischer Verbände in Deutschland im Juli 2007 am Zustandekommen des Integrationsgesetzes erwähnt werden, die übersah, dass das Parlament als Repräsentant des souveränen Volkes nicht verpflichtet werden kann, sein Gesetz von der Zustimmung außerparlamentarischer, zum Beispiel zivilgesellschaftlicher oder religiöser Organisationen abhängig zu machen. Ob die Vorgehensweise des Gesetzgebers klug war, steht auf einem anderen Blatt.

Die Ausübung politischer Macht ändert sich, gemessen am traditionellen Modell der Gewaltenteilung, durchaus substanziell. Im Netzwerk wird sie auf sehr viel mehr, Punkte ' verteilt, die Partizipation an der Macht wird breiter, im Einzelfall jedoch - womöglich - geringer.

In historischer Perspektive - es sei in Erinnerung gerufen, dass die Aufgabenstellung dieses Artikels , die historische Zukunft Europas“ lautet - macht es Sinn, die Zukunft der bisher erreichten und, wann immer es möglich ist, auch fürderhin ausbaubaren Einheit am Para-

21 Heinrich Schneider: Föderale Verfassungspolitik für eine Europäische Union, in: Heinrich Schneider/Wolfgang Wessels (Hrsg.): Föderale Union - Europas Zukunft? München 1994, S. 21-50, hier: S. 37.

22 Schneider: Föderale Verfassungspolitik, 1994, besonders S. 42-45.

23 Wolfgang Wessels: Die europäischen Staaten und ihre Union - Staatsbilder in der Diskussion, in: Schneider/ Wessels (Hrsg.): Föderale Union, 1994, S. 51-69, hier S. 61.

24 Vgl. z. B. Berthold Rittberger/Frank Schimmelfennig: Das Rätsel der Konstitutionalisierung. Wie aus der Europäischen Union ein Verfassungsstaat wurde, in: Berthold Rittberger/Frank Schimmelfennig (Hrsg.): Die Europäische Union auf dem Weg in den Verfassungsstaat, Frankfurt 2006, S. 15-39, hier zusammenfassend zu Kohärenz S. 35.

25 Grundlegend: Manuel Castells: The Rise of Network Society, Oxford 1996 (deutsch: Der Aufstieg der Netzwerkgesellschaft. Teil 1 der Trilogie Das Informationszeitalter, Opladen 2001). Castells setzt diesen ersten historisch-soziologischen Band in der Industriellen Revolution des 18. Jahrhunderts an. 
digma des ,Netzwerkes“ auszurichten. ${ }^{26}$ Der Netzwerkbegriff ist in der Debatte um das Innenleben der Europäischen Union präsent, bezieht sich in der Regel aber auf Interessensnetzwerke, die Lobbying betreiben oder sich zum Beispiel regional zusammenfinden, um regionalstrukturelle Ziele im wirtschaftlichen, politischen, kulturellen, technischen oder einem anderen Bereich zu verwirklichen. Der Begriff reicht aber sehr viel weiter als es der Alltagsgebrauch vermuten lässt. Bezieht man ihn auf Phänomene, die Heinrich Schneider als „hybrid“ charakterisierte, so ergibt sich folgendes Bild: Netzwerke verknüpfen explizit Akteure unterschiedlicher Art miteinander, sie zielen auf Kooperation und Ressourcenaustausch, auf die Realisierung unterschiedlichster Projekte materieller und immaterieller Art, sie zeichnen sich durch Stabilität, die jedoch zuerst aufgebaut werden muss, aus. Auf Seiten der Akteure ist keineswegs Homogenität erforderlich, ihre Zahl ist variabel. Netzwerke verbinden Variabilität mit Stabilität, das entspricht der Beförderung und Regulierung von Verflüssigung. Stabilität resultiert aus entwickeltem Vertrauen, aus Zuverlässigkeit und aus allseitigem Festhalten an gemeinsam gefundenen Verhaltensregeln, sie resultiert aus Partizipation, Dialog und Deliberation. Um es anders auszudrücken: Die Bereitschaft und Fähigkeit zu Vertrauen, zu Zuverlässigkeit, zum Respekt der gemeinsamen Regeln, die Anerkennung der Prinzipien von Partizipation, Dialog und Deliberativität sind letztlich im Sinne der Kopenhagener Kriterien als kongeniale Prinzipien von Demokratie, Rechtsstaatlichkeit und Schutz der Grund- und Menschenrechte anzusehen. Diese Prinzipien kennzeichnen essentiell, was als Netzwerkmethode bezeichnet werden kann. Wendet man sie auf die konfliktreichen Beziehungen wie Europäische Union - Russland und andere an, erweist sich, dass die in diesen Fällen derzeit strukturelle Nichtanwendbarkeit den Unterschied zu jenen Beziehungen ausmacht, wo eine strukturelle Anwendbarkeit besteht. Die genannten Prinzipien setzen - wie Rechtsstaatlichkeit, Demokratie, Menschenrechte - nicht nur politische, sondern auch kulturelle und soziale Kompetenzen bei den Akteuren voraus. Ohne deren vorherige Entwicklung und Aneignung besteht keine Möglichkeit, Akteur im UnionsNetzwerk zu werden.

Der Netzwerkbegriff lässt sich weder auf das Netzwerk von Lobbyisten oder Interessenvereinigungen noch allein auf policy networks reduzieren. Sein Potenzial wird durch die breite Anwendung in der Soziologie, Politikwissenschaft oder Geschichtswissenschaft angezeigt. Offenkundig handelt es sich um einen vielseitig einsetzbaren Begriff, der auf das Zusammenwachsen von Gesellschaft (Zivilgesellschaft), Staat, Politik und Wirtschaft reagiert. Wenn die aufgezählten Wissenschaften ihn verschieden einsetzen, so liegt dies daran, dass sie die Wirklichkeit aus sehr verschiedenen Blickwinkeln betrachten. Verschiedene Blickwinkel nützen aber letztlich nur dann etwas, wenn es gelingt, sie in einer Synthese zusammenzuführen.

Die synthetischen Qualitäten des Begriffs erweisen sich, wenn ein sogenanntes Defizit der Europäischen Union in den Blick genommen wird: der fehlende europäische Demos und die fehlende europäische Öffentlichkeit. Beides gehört eng zusammen. Zusammenhänge wie die politisch-demokratische Bedeutung von Demos und Öffentlichkeit können hier als bekannt vorausgesetzt werden. Bevor ein europäischer Demos und eine europäische Öffentlichkeit entstehen können, bedarf es der Vernetzung der Menschen in Europa zu einem Kommunikationsnetzwerk und zu einer europäischen Gesellschaft, für die die Identifikation mit

26 In diese Richtung zielt ein Vorschlag von Philip Cooke, der wesentliche definitorische Elemente für Netzwerk an Hand von ,policy-Netzwerken“ (Cooke's Schreibweise) gewinnt, auf die ich hier zurückgreife: Philip Cooke: Policy-Netzwerke, Innovationsnetzwerke und Regionalpolitik, in: Hubert Heinelt (Hrsg.): Politiknetzwerke und europäische Strukturfondsförderung. Ein Vergleich zwischen EU-Mitgliedstaaten, Opladen 1996, S. 58-74, hier besonders S. 64. 
der Union Sinn macht und Funktionalität besitzt. ,Vernetzung zu europäischer Gesellschaft beinhaltet mehr als nur Vernetzung zu einer Kommunikationsgemeinschaft. Dieser Prozess ist in Gang gesetzt; er wird nicht nur durch die Vernetzung von Individuen, nicht zuletzt durch EU-Programme, sondern auch durch verschiedene zivilgesellschaftliche Institutionen unterstützt. Auch die geschaffene Unionsbürgerschaft und die unmittelbar damit verknüpften Wahlrechte unterstützen den Prozess, der eher am Anfang, denn in der Mitte steht. Einstweilen macht es Sinn, auch auf der institutionellen Ebene auf der Grundlage des erreichten Einheitsstandes die Europäische Union mit der Netzwerkmethode fortzuentwickeln.

Es geht nicht um revolutionär Neues, vielmehr geht es darum, sich klar zu machen, dass bestimmte längst eingeleitete Entwicklungen einen Paradigmenwechsel bedeuten. Eine Option der Netzwerkmethode besteht darin, statt in den Kategorien Vollmitgliedschaft, ,privilegierte Partnerschaft' oder Assoziation zu denken, die Möglichkeit sehr viel feingliedrigerer Kategorien von Akteuren auszuloten, deren Ziel die Etablierung von Kohärenz auf einem bestimmten Feld ist. Gemeint ist die Beteiligung unterschiedlicher Länder oder Regionen an unterschiedlichen Handlungsfeldern der Europäischen Union in unterschiedlichem Ausmaß. Faktisch geschieht dies bereits mit unterschiedlichem Erfolg: im Falle Russlands und der Zusammenarbeit auf dem Energiesektor stockt der Prozess, im Fall Brasiliens schreitet er voran, im Fall der Kaukasus- und Zentralasiatischen Staaten wird man sehen. Wenn der Kosovo in der Obhut der Vereinten Nationen selbständig wird, wird die Europäische Union Angebote machen müssen, bei denen weder Serbien noch Albanien noch die Ehemalige jugoslawische Republik Mazedonien außerhalb der Betrachtung bleiben können. Allen vier die Vollmitgliedschaft anzubieten, ist derzeit nicht denkbar, nur einem oder zwei der vier sie anzubieten, ist ebenso wenig denkbar, weil damit keine Konflikte gelöst, sondern nur verschärft würden. In dieser Situation könnte es hilfreich sein, die Netzwerkmethode zu einem offiziellen Instrument der Europäischen Union gemacht zu haben. Die Anwendung des Netzwerk-Paradigmas ist ,egalitärer" als verschiedene Typen von Partnerschaft definieren zu wollen, die immer Hierarchien und Asymmetrien betonen. Der - beispielsweise - Türkei statt der Vollmitgliedschaft eine privilegierte Partnerschaft anzubieten, heißt Asymmetrie und Hierarchie besonders krass auszudrücken und damit Hindernisse auf- statt abzubauen.

Unionsmitglieder können nur Staaten sein, während Subeinheiten von Staaten wie Regionen, Provinzen, Bundesländer etc., aber auch Kommunen (Großstädte) durchaus Akteure im europäischen Netzwerk sein können. Dieser Aspekt entfaltet seine Bedeutung in Bezug auf alle jene Staaten (wie etwa Russland), die als Ganzes kaum Mitglied der Union werden können oder wollen, während der Status eines Netzwerk-Akteurs in der Union für regionale Einheiten oder bestimmte Städte, je nach Ausmaß ihrer verfassungsmäßigen Rechte in ihrem Staat vorstellbar ist. Ja, mehr noch: es wäre denkbar, die Ausgestaltung von regionalen Autonomien oder jedenfalls verfassungsmäßigen Rechten analog zu den Bundesländern in europäischen Bundesstaaten im Hinblick auf die Möglichkeit, Netzwerk-Akteur der Europäischen Union zu werden, voranzutreiben.

Die Fortentwicklung der Europäischen Union mittels der Netzwerkmethode muss sich nicht auf den europäischen Raum beschränken, während der Methode des Mitgliedschaftserwerbs sehr viel engere Grenzen gesetzt sind. Bisher galt es als erstrebenswertes Ziel, die drei Europäischen Gemeinschaften zu einer Gemeinschaft im Singular zu verschmelzen. Es scheint sich jedoch als pragmatisch herauszustellen, wieder mehr an Gemeinschaften zu denken, die im engeren Wortsinn miteinander zu vernetzen sind, die also nicht als sektorale Gemeinschaften, wie es anfangs der Fall mit den drei Gemeinschaften von EGKS, EWG und Euratom gewesen war, vorzustellen sind. Die Knoten, in denen die Fäden zusammenlaufen und die Kohärenzerzeugung, überwacht ‘ wird, und die dafür sorgen, dass die Europä- 
ische Union ein Sinnganzes bleibt, sind geschaffen: Europäisches Parlament, Europäische Kommission, Ministerrat, Europäischer Rat, Europäischer Gerichtshof.

Was in diesem Zusammenhang realisiert werden sollte, ist der Umstand, dass der Paradigmenwechsel bereits im Gange ist. Ein keineswegs kleiner Kern von Einheit, der verfassungsmäßig gesichert ist, besteht; hier kann das Paradigma des Netzwerkes ansetzen und methodisch ausgebaut werden. Man darf sich nicht erwarten, dass die Europäische Union der Zukunft in einer „Mini-Verfassung“, wie es der französische Staatspräsident Sarkozy gefordert hatte - bevor er aus der Mini-Verfassung im Wahlkampf einen „Mini-Vertrag“ machte - bestehen wird. Nirgendwo, weder auf der Ebene des Individuums, der Gesellschaft, der nationalen politischen Systeme, weder auf der Ebene der Bildungs- und Wissenschaftssysteme, weder auf der Ebene der Wirtschaft, noch der Kultur, noch der Religion werden die Verhältnisse einfacher und transparenter, sie werden alle komplexer, sie pluralisieren sich. Das historische Paradigma der Einheit stößt angesichts dieser globalen Entwicklung an seine Leistungsgrenzen. Der Europäischen Union kann es in dieser Umwelt nicht anders ergehen. Entscheidend ist, die Komplexität und Pluralisierung nicht zu erleiden, sondern sie zu durchdenken und zu gestalten. Mir scheint das Paradigma des Netzwerks der geeignete Ansatzpunkt, um die , historische Zukunft Europas‘ zu denken.

\section{Neu im Programm}

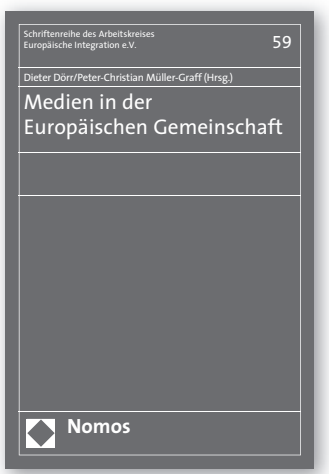

\section{Medien in der Europäischen Gemeinschaft}

Herausgegeben von Dieter Dörr und

Peter-Christian Müller-Graff

2007, 158 S., brosch., 29,- €, ISBN 978-3-8329-3128-5

(Schriftenreihe des Arbeitskreises Europäische Integration e.V., $B d .59)$

Die Frage nach der Rolle der Medien und transnationalen Kommunikation war 2006 Gegenstand einer interdisziplinären Tagung des Arbeitskreises Europäische Integration in Zusammenarbeit mit der Universität Mainz. Der vorliegende Band enthält zehn zur Veröffentlichung bearbeitete Vorträge. 